\title{
Non-Operative Management of Blunt Solid Abdominal Organ Injury in Calabar, Nigeria
}

\author{
Asuquo Maurice ${ }^{1}$, Bassey Okon ${ }^{1}$, Etiuma Anietimfon ${ }^{1}$, Ngim Ogbu ${ }^{1}$, Ugare Gabriel $^{1}$, Anthonia Ikpeme ${ }^{2}$ \\ ${ }^{1}$ Departments of Surgery, University of Calabar Teaching Hospital, Calabar, Nigeria; ${ }^{2}$ Departments of Radiology, University of Calabar \\ Teaching Hospital, Calabar, Nigeria. \\ Email: \{mauefas, odokwobassey, ogbungim, udeyhenugare, iaikpeme\}@yahoo.com, aetiuma@yahoo.co.uk
}

Received August $9^{\text {th }}, 2010$; revised August 10 $0^{\text {th }}, 2010$; accepted August $12^{\text {th }}, 2010$.

\begin{abstract}
Background: Over the past several years, non-operative management has been increasingly recommended for the care of selected blunt abdominal trauma patients with solid organ injuries. Objective: To evaluate the pattern and outcome of blunt abdominal trauma using haemodynamic stability and ultrasonography in the selection of patients for non-operative management in a facility without computed tomogram. Methods: Patients admitted with blunt abdominal trauma between February 2005 and January 2010 were prospectively studied. Haemodynamic stability and sonography formed the basis for selecting patients for non-operative management. Results: In total, 58 patients suffered blunt abdominal trauma and 19(33\%) patients were successfully managed non-operatively suffered blunt solid abdominal organ injuries. Road traffic accidents inflicted $17(89 \%)$ patients while 2(11\%) patients sustained sports injury (football). The spleen was the commonest solid organ injured 12(60\%), while the liver and kidney were injured in 6(30\%) and 2(10\%) respectively. Associated injuries were fractured left femur recorded in 3(16\%) patients and fractured rib in a patient (5\%). Conclusion: Nonoperative treatment is a safe and effective method in the management of haemodynamically stable patient with blunt solid abdominal organ injury. This translated to a reduction in hospital stay, absence of the risk of blood transfusion as well as attendant morbidity and mortality associated with laparotomy. Establishment of trauma system, provision of diagnostic and monitoring facilities, good roads, and education on road safety is recommended for improved outcome.
\end{abstract}

Keywords: Blunt Abdominal Trauma, Solid Organ Injury, Non-Operative Management

\section{Introduction}

The management of patients with blunt abdominal trauma (BAT) has evolved over the past two decades with increase reliance on a non-operative approach $[1,2]$. During the past decades, non-operative management (NOM) of haemodynamically stable blunt trauma patients with liver, spleen has become the standard of care [3]. The increased utilization of nonoperative or conservative surgical management of abdominal solid organ injuries is facilitated by various sophisticated or highly accurate noninvasive imaging tools at the trauma surgeons disposal [2]. Its ability to demonstrate the amount of intra-peritoneal haemorrhage within minutes of patients arrival, sonography may be considered the screening modality of choice for BAT [4].

Ultrasound is used worldwide to evaluate patients with BAT and sometimes referred to as an extension of physical examination as it is portable, integrates easily into the resuscitation of trauma victims, non invasive and has no associated morbidity [5]. The reliance of haemoperitoneum as the sole indicator of abdominal visceral injury limits the utility of focused abdominal sonography for trauma (FAST) [2].

We undertook a prospective study of abdominal trauma as part of a wider study of the Trauma Research group. This 5-year report sought to evaluate the pattern and outcome of NOM of blunt abdominal solid organ injury in our setting with poor patients and limited facilities for emergency care.

\section{Materials and Methods}

Patients admitted into the University of Calabar Teaching Hospital (UCTH), Calabar, Nigeria with BAT from February 2005 through January 2010 were prospectively studied. This included biographic data, mechanism, and pattern of injuries including associated injuries. Ultrasonography and other investigations included.

Ultrasonography findings, haemodynamic stability in 
the absence of computerized tomography (CT) in our facility formed the basis for selection of patients for NOM. Ultrasonography finding of haemoperitoneum were classified into mild, moderate, and severe. The amount of haemoperitoneum was quantified as follows: mild (minimal) - blood in the subphrenic or perihepatic spaces (approximately $500 \mathrm{ml}$ ); moderate-perihepatic and blood along paracolic gutter (less than $1 \mathrm{~L}$ ); and large (severe) perihepatic, blood along the paracolic gutter and accumulation in the pelvic cavity (more than $1 \mathrm{~L}$ ) [6].

Radiographic studies of the abdomen (plain X-ray) are indicated in stable patients when physical examination findings are inconclusive [7].

The total number of patients enrolled for NOM with solid organ injury was compared with total number of patients with BAT seen during the same period of study.

\section{Results}

A total of 58 patients suffered BAT, 19(33\%) patients with solid abdominal organ injury were managed non-operatively, and another patient managed non-operatively suffered mesenteric vascular injury, while 38 had laparotomy.

The ages of the patients ranged from 16 through 48 years (mean 27 years). The sex distribution revealed 13 males and 6 females $(\mathrm{M}: \mathrm{F}=2.2: 1)$

Table 1 shows the mechanism of injury, road traffic accident (RTA) in $17(89 \%)$ patients while $2(11 \%)$ patients suffered injury from sports (football). Of the RTA, 4 were motor vehicle (MV) related and 13 motorcycle (MC). The spleen was the commonest solid organ injured $12(60 \%)$, Figure 1, others were, liver 6(30\%), and renal contusion (With evidence of retroperitoneal haematoma), 2(10\%). A patient (MV-occupant) suffered multiple organ injury (liver/spleen), while 3 patients (MC/pedestrian) suffered splenic injuries associated with fractured left femur, Table 1. The mean injury severity score (ISS) was 7.9. In the laparotomy group (38), 37(97\%) were RTA and one kick on the abdomen (football). A total of 43 abdominal organ and 14 associated injuries were recorded (Head injury 6, Fractures 7, Spinal injury 1). Four patients suffered multiple abdominal organ injuries, and 12 were polytraumatized. The mean ISS was 12.5 .

The time from incident to admission in the NOM group ranged between 30 mins and 4 hrs (mean $1.8 \mathrm{hrs}$ ), while that of the laparotomy group ranged between 15 mins and $4 \mathrm{hrs}$ (mean $1.9 \mathrm{hrs}$ ). Sonography was the main tool for patient triage in our study. Time from admission to sonography ranged between $1 \mathrm{hr}$ and $3 \mathrm{hrs}$ (mean 1.64 hrs). Immediate post treatment scan done prior to discharge was to ensure absence of ongoing bleeding (Figure 2) and 4 weeks post injury to access healing and absorption of haemoperitoneum. Weekly reviews were done on outpatient basis for 1 month and monthly for 2 months. Six patients $(31.0 \%)$ had mild haemoperitoneum, $10(53.0 \%)$ moderate and $3(16 \%)$ severe haemoperitoneum (Figure 3).

The blood pressure and pulse was measured $1 / 4$ hourly initially until patients were stable. The systemic pressure in the NOM group averaged $110 \mathrm{mmHg}$ when compared to $80 \mathrm{mmHg}$ in the laparotomy group. The average haematocrit in the NOM group was $33 \%$ compared to $22 \%$ in the laparotomy group. The average Glasgow coma scale (GCS) in the NOM was 15 while that of the laparotomy was 14.2. All the patients were mobilized by the third day post trauma (excluding patients with associated lower limb fracture) and the mean duration of admission in our service was 6.9 days when compared to 12.3 days in the laparotomy group. This was statistically significant (p-value - 0.000). Patients with severe haemoperitoneum had parenteral iron while others were given oral iron tablets. No patient had blood transfused. Co-morbidity was fractured femur observed in 3 patients the NOM group including one with fractured rib. There was no hospital mortality in both groups.

Table 1. Blunt abdominal solid organ injury

\begin{tabular}{|c|c|c|c|c|c|}
\hline Mechanism of injury & No. $(\%)$ & Organ injury & No. $(\%)$ & Associated injury & No. $(\%)$ \\
\hline Road traffic injuries & & Spleen & $12(60)$ & Fracture (L) femur & $3(16)$ \\
\hline Motorvehicle (MV)-occupant & $3(16)$ & Liver & $6(30)$ & Fracture rib & $1(5)$ \\
\hline $\mathrm{MV} /$ pedestrian & $1(5)$ & Renal Contusion & $2(10)$ & & \\
\hline Motorcycle (MC)-cyclist & $4(21)$ & & & & \\
\hline $\mathrm{MC} /$ pedestrian & $9(47)$ & & & & \\
\hline \multicolumn{6}{|l|}{ Sports injury } \\
\hline Kick on the abdomen (Football) & $2(11)$ & & & & \\
\hline Total & $19(100)$ & & $20(100)$ & & \\
\hline
\end{tabular}

*One patient (MV occupant) suffered multiple organ injury (liver/spleen).

*Two patients (MC/pedestrian) suffered splenic injuries associated with \# (L) femur.

*One patient (MC/Pedestrian) suffered \# rib. 


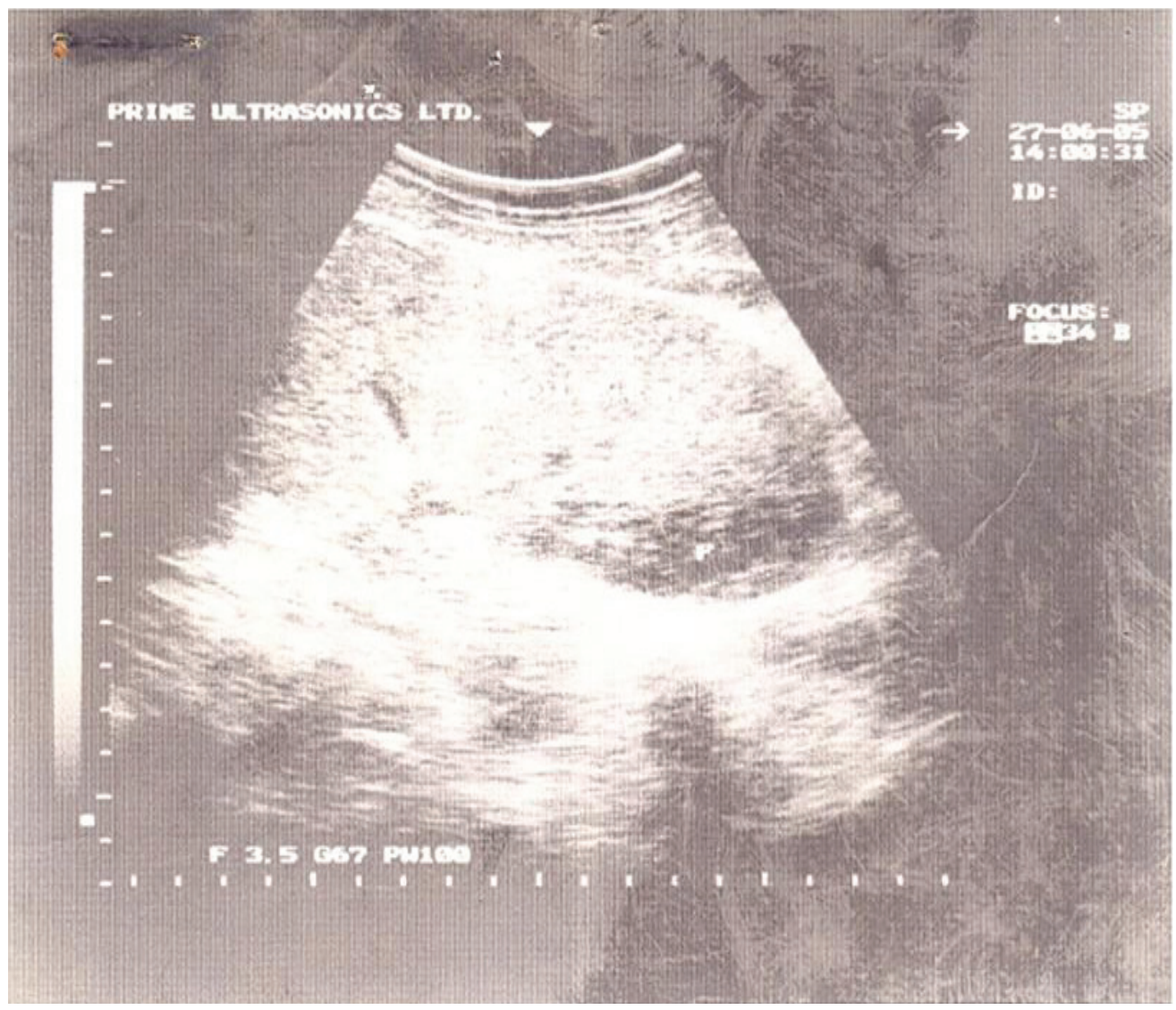

Figure 1. Showing splenomegally with subcapsular haematoma from splenic contusion (MC/pedestrian collision)
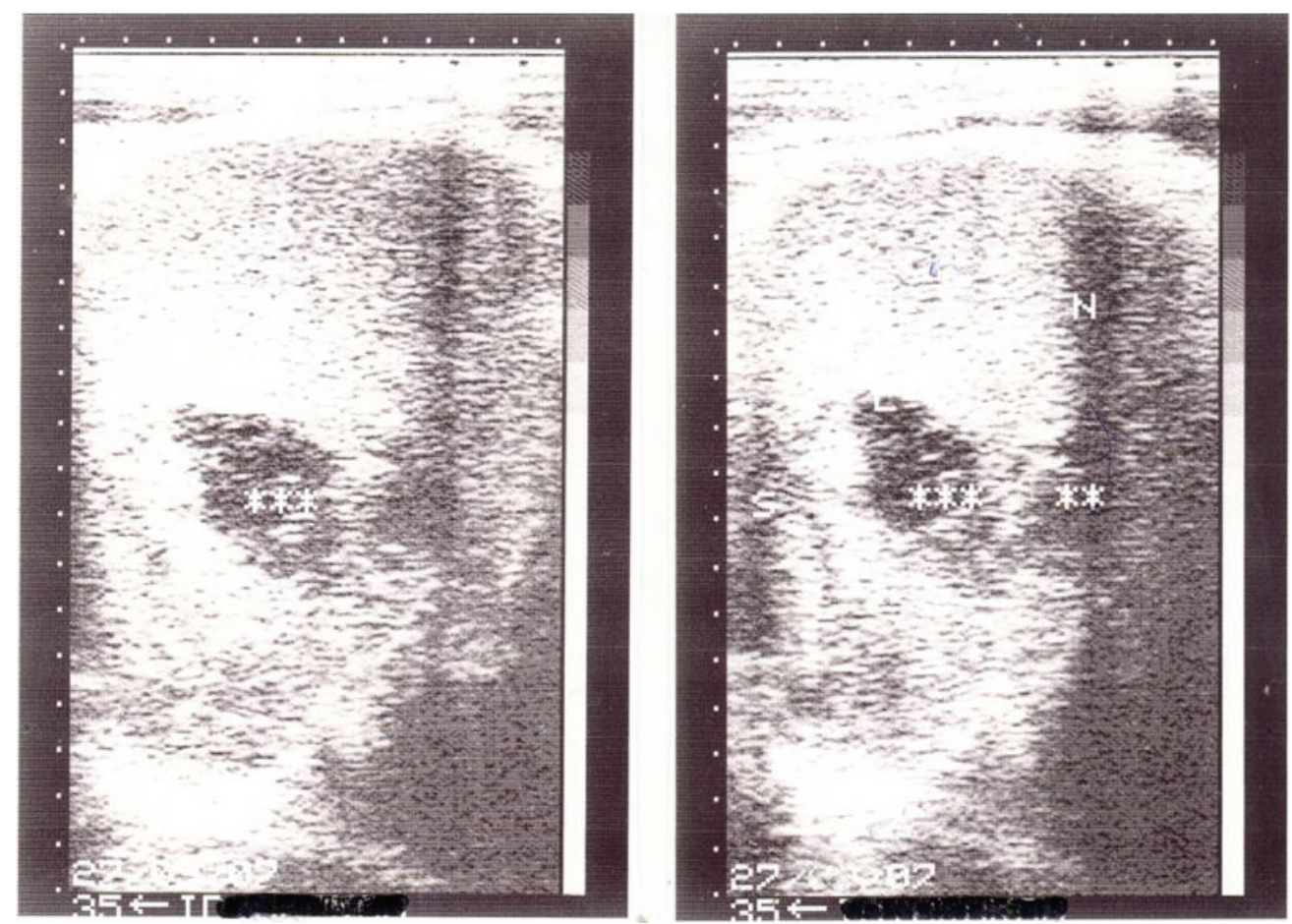

Figure 2. Traumatic splenic rupture - (repeat USS prior to discharge) 


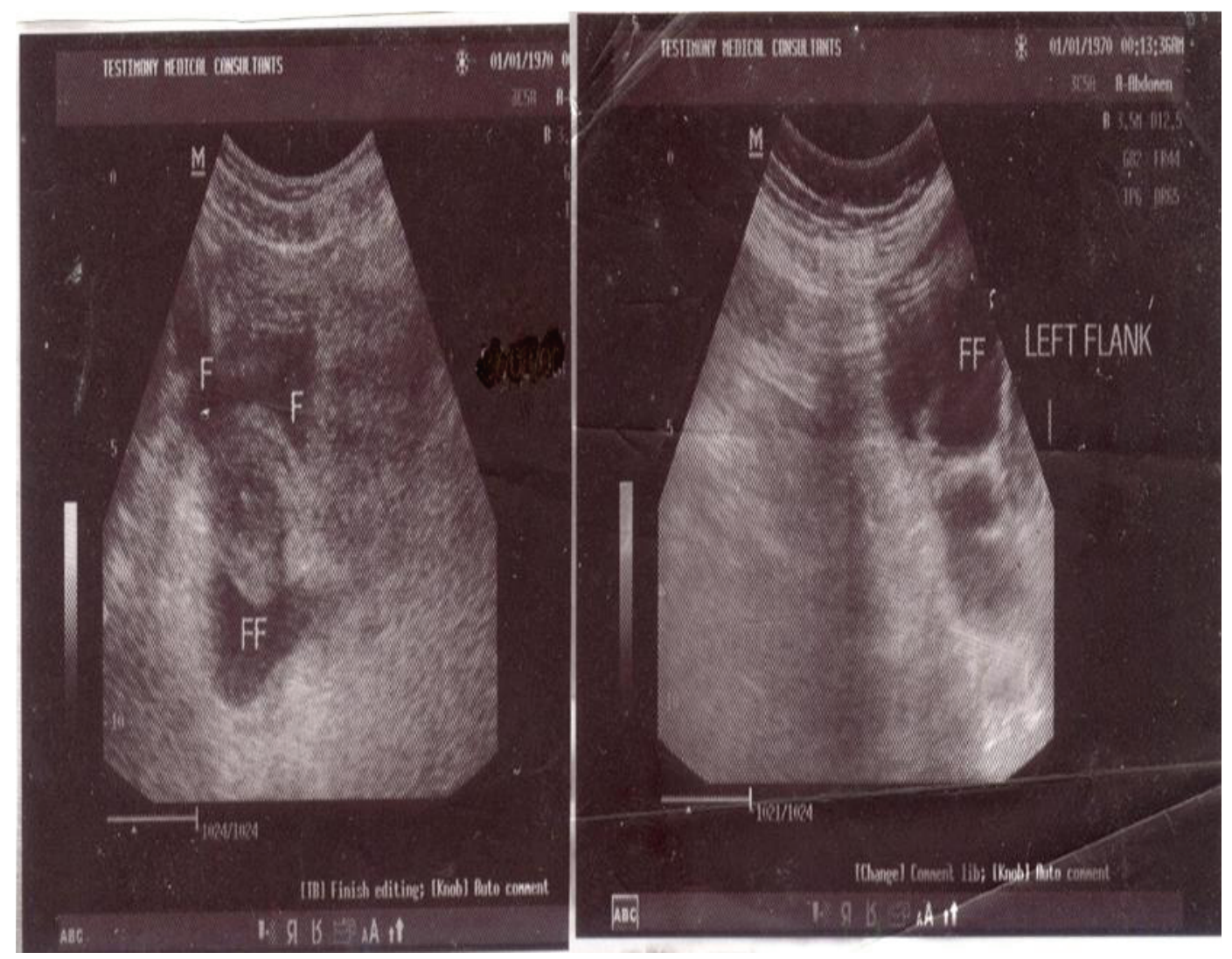

Figure 3. Free peritoneal fluid collection - Severe haemoperitoneum

\section{Discussion}

Nineteen patients with solid abdominal injuries were managed successfully under the NOM group. In Calabar, there is no ambulance and paramedic service; hence, prehospital intervention is not in place. In addition, there is no proper trauma system. Doctors and nurses trained in emergency medicine receive the injured at the emergency department (ED) for resuscitation and primary survey. Subsequently, patients were referred to the appropriate surgical unit on call based on the injury. The first on call is the registrar followed by the senior registrar and the consultant surgeon. Anaesthesiology and Radiology are also organized in a similar pattern. A theatre is dedicated for emergencies with a 3-bed intensive care unit. An understanding of the clinical and radiographic parameters used to determine those who may be eligible for this form of treatment is an essential component of modern trauma care [1]. Haemodynamic stability was defined as serial blood pressure and heart rate appropriate for age with adequate end organ perfusion measured on admission or after fluid resuscitation with normal saline/Ringers lactate. Clinical, ultrasonography and laboratory evidence of ongoing hemorrhage or development of peritonitis-determined failure of NOM. Ongoing hemorrhage was defined clinically as progressive abdominal distension (measured initially half hourly). Also tachycardia (pulse rate $>100 / \mathrm{min}$ ) or hypotension (systolic pressure $<90 \mathrm{mmHg}$ ) refractory to fluid resuscitation with a urinary output of $<30 \mathrm{mls} / \mathrm{hr}$. Progressive fall in packed cell volume (PCV) and ultrasonography evidence of ongoing bleeding were other indicators for failure of NOM. Haemodynamic stability and ultrasonography effectively selected patients for conservative management in our setting without a CT. This is significant in our setting where health funding is poor as well as poor patients. In our report, $33 \%$ of BAT patients were managed nonoperatively. Norman et al. reported that $55 \%$ were managed conservatively with a low failure rate and routine CT scan did not appear to add clinically relevant information affecting patient management. [8] Other reports were; Yaghoubi et al. 31.5\% [9], Al-Mulhim and Mohammed $82.5 \%$ [9], Velmathos et al. 85\% [10], however, these centers had CT. Sonography has been shown to be comparable with diagnostic peritoneal lavage and CT for the detection of haemoperitoneum and superior to both modalities because of its rapidity, non-invasiveness, portability, and low cost [4]. However, solid organ injuries without haemoperitoneum could be missed; in addition, 
it is not as efficient as CT in characterization of injuries. Chiu et al. reported that $15 / 52$ (29\%) patients with abdominal injury had no haemoperitoneum thereby limiting the utilization of FAST [11].

BAT affected mainly young patients (mean age 27.0 years). This is in keeping with an earlier report from this centre and other centers in Nigeria [12-14]. They constitute an active and productive segment of the society with greater mobility with increased risk of accident. There was a male preponderance $(\mathrm{M}: \mathrm{F}=2.2: 1)$, studies have shown that men tend to be affected slightly more than women are [7].

BAT usually results from MV collisions, assault, recreational accident, or falls [7].

Vehicular trauma is by far the leading cause of BAT in civilian population, auto to auto and auto to pedestrian collisions have been cited as causes of $50-75 \%$ of cases. [6] This report reveals a pattern peculiar to our setting. RTA accounted for $89 \%$ of injuries while $11 \%$ were due to recreational injury (football). Majority of the road traffic injuries were due to $\mathrm{MC} /$ pedestrian collisions. In our setting motorcycles have become a popular means of transportation due to poor road infrastructure. Motorcycles are distributed as reward for political patronage to youths who are inexperience, and sometimes drunk. Good road construction, less use of motorcycles would reduce the incidence of road traffic accidents.

The spleen was the commonest solid organ injured. Treatment of blunt splenic trauma has undergone dramatic changes over the last few decades. NOM is now the preferred treatment of choice when possible [8]. This is particularly important as we recorded these injuries in young patients; other studies recommend conservation especially in young stable patients [9]. The liver ranked second of solid organ injury. NOM for hepatic and splenic injuries have been well documented $[6,15]$.

Risks associated in NOM are missed injuries, delayed diagnosis and treatment, retained haematoma, sepsis and or abscess [16]. A female patient earlier enrolled for NOM had laparotomy because of peritonism diagnosed on the $5^{\text {th }}$ day post trauma. This patient had evidence of pelvic inflammatory disease prior to the trauma, which proved to be a contraindication for NOM. In a study by Bimar et al., they reported a failure rate of $3 \%$ due to rebleeding from grade iv liver injury [17]. Peitzman et al. reported that $30-40 \%$ of the patients initially scheduled for Nonoperative management were unsuccessful because they were selected inappropriately with haemodynamic instability and initial misinterpretation of diagnostic study [18]. Malhotra et al. [19], and Schroeppel and Croce [20] reported that nonoperative management significantly improved outcomes over operative managements in terms of decreased abdominal infections, decreased transfusions and decreased lengths of stay. This was in keeping with our findings.
All our patients were mobilized by the third day post trauma in the absence of associated injuries limiting movement. Boggs found that the timing of mobilization of patients with blunt solid organ injury was not associated with a higher incidence of delayed rupture. Protocols that incorporate a period of strict bed rest are unjustified and may contribute to morbidity, longer hospital stay, and higher hospital cost [21].

None of our patients required plain abdominal X-ray, as the abdominal findings in all our patients were conclusive. Although, the overall value of plain films in the evaluation of patients with BAT is limited, they can demonstrate numerous findings; chest radiographs may aid in diagnosis of ruptured hemi diaphragm or pneumoperitoneum, pelvic or chest radiographs can demonstrate fractures of the thoraco-lumbar spine and free intraperitoneal air, or trapped retroperitoneal air from the duodenal perforation [7].

The mean ISS were 7.9 and 12.5 for the nonoperative and laparotomy groups respectively indicating that patients in the laparotomy group suffered more severe injuries. However, ISS may be of limited clinical value when considering any individual case. Al Mulhim and Mohammed reported that judgment to operate or not was dependent mainly on the clinical parameters rather than numerical values of ISS [10].

\section{Conclusions}

Nonoperative management is safe and effective method in the management of blunt solid abdominal organ injury in haemodynamically stable patient. Ultrasonography was reliable in diagnosis and monitoring of patients in the absence of CT. The benefits were decreased morbidity and mortality associated with laparotomy, decreased blood transfusion and decreased length of hospital stay. A reduction in RTA by the provision good and well-designed roads and traffic infrastructure, health education on road safety would improve outcomes with a reduction in the health care cost in trauma management.

\section{REFERENCES}

[1] D. E. Gibson, C. M. Canfield and P. D. Levy, "Selective Non-Operative Management of Blunt Abdominal Trauma," Journal of Emergency Medicine, Vol. 31, No. 2, 2006, pp. 215-221.

[2] S. P. Stawicki, "Trends in Nonoperative Management of Traumatic Injuries," OPUS 12 Scientist, Vol. 1, No. 1, 2007, pp. 19-35.

[3] P. R. Miller, M. A. Croce, T. K. Bee, A. K. Malhotra and T. C. Falran, "Associated Injuries in Blunt Solid Organ Trauma: Implication for Missed Injury in Non-Operative Management," Journal of Trauma, Vol. 53, No. 2, 2002, pp. 238-242.

[4] K. L. McKenny, "Ultrasound of Blunt Trauma," Radiologic Clinics of North America, Vol. 37, No. 5, 1999, 
pp. 879-893.

[5] M. A. Brown, C. B. Sirlin, D. B. Hoyt and G. Casola, "Screening Ultrasound in Blunt Abdominal Trauma," Journal of Intensive Care Medicine, Vol. 18, No. 5, 2003, pp. 253-260.

[6] Y. G. Goan, M. S. Huang and L. Jin, "Non Operative Management of Extensive Hepatic and Splenic Injuries with Significant Haemoperitoneum in Adults," Trauma, Vol. 45, No. 2, 1998, pp. 360-365.

[7] J. Udeani and S. R. Steinberg, "Abdominal Trauma: Blunt," eMedicine Journal, 2006. http://med.javeriana. edu.co/pediatria/revis/eMedicineudeani.htm

[8] G. Norman, B. Tingstedt, M. Ekelund and R. Andersson, "Nonoperative Management of Blunt Splenic Trauma: Also Feasible and Safe in Centres with Low Trauma Incidence and in the Presence of Established Risk Factors," European Journal of Trauma and Emergency Surgery, Vol. 35, No. 2, 2009, pp. 102-107.

[9] A. Y. Yaghoubi Notash, H. A. Ahmadi Amoh, A. Nikandish, A. Yazdankhah Keran, F. Jahangiri and P. Khashayer, "Non-Operative Management of Blunt Splenic Trauma," Emergency Medicine Journal, Vol. 25, No. 4, 2008, pp. 210-212.

[10] G. C. Velmahos, K. Toutouzas, R. Radin, L. Chan, P. Rhee, A. Tillou and D. Demetriades, "High Success with Non-Operative Management of Blunt Hepatic Trauma," Archives of Surgery, Vol. 138, No. 5, 2003, pp. 475-481.

[11] W. C. Chiu, B. M. Cushing, A. Rodriguez, S. H. Ho, S. E. Mirvis, K. Shanmuga Nathan and M. Stein, "Abdominal Injuries without Haemoperitoneum a Potential Limitation of Focused Abdominal Sonography for Trauma (FAST)," Journal of Trauma, Vol. 42, No. 4, 1997, pp. 617-623.

[12] M. E. Asuquo, V. Nwagbara, A. Inyang and G. Ugare, "Blunt Abdominal Trauma in Calabar," Nigeria Journal of Surgical Science, Vol. 16, No. 3, 2006, pp. 12-15.

[13] M. N. Okobia and U. Osime, "Abdominal Trauma in
Benin, Southern Nigeria," Nigeria Journal of Surgery, Vol. 5, No. 2, 1998, pp. 49-54.

[14] D. Dogo, T. Yawe, A. W. Hassan and B. Tahir, "Pattern of Abdominal Trauma in North-Eastern Nigeria," Nigeria Journal of Surgical Research, Vol. 2, No. 2, 2000, pp. 48-51.

[15] A. S. Al-Mulhim and H. A. H. Mohammed, "Non Operative Management of Blunt Hepatic Injury in Multiple Injured Adult Patients," Journal of Royal College of Surgeons in Ireland, Vol. 1, No. 2, 2003, pp. 81-85.

[16] S. Gopalswamy, R. Mohanraj, P. Viswanathan and V. Biskaran, "Nonoperative Management of Solid Organ Injuries due to Blunt Abdominal Trauma (NOMAT). Seven-Year Experience in a Teaching District General Hospital - A Prospective Study," Internet Journal of Surgery, Vol. 15, No. 2, 2008.

[17] H. A. Bismar, M. K. Alam, M. H. Al-Keely, S. M. Al Salamah and A. A. Mohammed, "Outcome of Nonoperative Management of Blunt Liver Trauma," Saudi Medical Journal, Vol. 25, No. 3, 2004, pp. 294-298.

[18] A. B. Peitzman, B. G. Harbrecht, L. Rivera and B. Heil, "Failure of Observation of Blunt Splenic Injury in Adults: Variability in Practice and Adverse Consequences," Journal of the American College of Surgeons, Vol. 201, No. 2, 2005, pp. 179-187.

[19] A. K. Malhotra, T. C. Fabian, M. A. Croce, T. J. Gavin, K. A. Kudsk, G. Minard and F. E. Pritchard, "Blunt Hepatic Injury: Paradigm Shift from Operative to Nonoperative Management in the 1990s," Annals of Surgery, Vol. 231, No. 6, 2000, pp. 804-813.

[20] T. J. Schroeppel and M. A. Croce, "Diagnosis and Management of Blunt Abdominal Solid Organ Injury," Current Opinion in Critical Care, Vol. 13, No. 4, 2007, pp. 399-404.

[21] W. Boggs, "Early Mobilization Safe for Patients with Blunt Solid Organ Injuries," Archives of Surgery, Vol. 143, No. 10, 2008, pp. 972-976. 